\title{
Los Fonemas del Inga
}

Por

JEAN CAUDMONT 


\section{SUMARIO}

1.-Localización de los Indios Inganos.

2.-Clasificación del Inga.

3.-Los Fonemas.

4.-Definición de los fonemas.

5.-Realización de los fonemas.

6.-Acentuación.

7.-Estructura de la sílaba.

8.-Distribución de los fonemas.

9.-Conclusiones. 
1.-El Inga ( /inka/ ), idioma de los indios Inganos ( /inkanu/ ), se habla en la mayor parte de la Comisaría del Putumayo, en el sur de la República de Colombia. (1)

Los Indios Inganos viven en los alrededores de la carretera que va de Pasto a Mocoa y atraviesa la Comisaría de Este a Oeste; aparecen desde la laguna de La Cocha, al norte y al sur de la carretera, excepto en el pueblo de Sibundoy ocupado por los Indios Kamsa (2); al Este se encuentran más allá de Puerto Limón, en las riberas del río Caquetá; al Norte alcanzan a Descance sobre el mismo río y al Sur casi hasta Wiskey sobre el río Putumayo.

La descripción fonológica que se presenta a continuación se basa en el Inga tal como se habla en el pueblo de Santiago y sus alrededores, o sea en la parte más occidental del grupo Ingano, entre los indios en estado más avanzado de aculturación.

Todos los Inganos son hoy día bilingües (inga y español) y a veces trilingües (inga, kamsa y español). Los individuos de ambos sexos aprenden a hablar castellano desde la infancia y usan este idioma de manera satisfactoria a pesar de algunos arcaismos y quichuismos usuales en la región y en las zonas vecinas. Los indios Inganos son, sin embargo, todavía analfabetas en su casi totalidad.

El Inga presenta, de manera evidente, interferencias del sistema fonológico del español local y de su vocabulario; asimiló numerosas palabras españolas sin que hoy día los indígenas se dieran cuenta de que son préstamos de su segundo idioma. No trataremos aquí de la estructura gramatical ni del vocabulario de esta lengua que reservamos para futuras publicaciones. Tampoco trataremos de la fonología diacrónica que estudiaremos en un próximo artículo donde ensayaremos explicar la evolución

(1) Cf. Milcíades Chaves Ch. La Colonización de la Comisaría del Putumayo: un Problema etno-económico-geográfico. En: Boletín de Arqueología, Año I, No 6, Bogotá, 1945.

(2) Los Inganos les llaman /kuči/. Hablan un idioma completamente distinto. 
del sistema fonológico quichua por influencia del sistema fonológico español.

Nuestro principal informante ha sido Domingo Tandioy, de unos 50 años de edad y oriundo de Santiago, cuyo conocimiento del castellano era bastante bueno y que además sabía algo de Kamsa, pero sin que el conocimiento de este último idioma sea suficiente para influír de manera alguna sobre su pronunciación del Inga. (3).

2.-El Inga es un dialecto de la lengua Quichua, que se habla desde el sur de Colombia hasta el norte de la Argentina (Provincia de Tucumán) y que comprende la mayor parte de las tierras altas del Ecuador, del Perú y de Bolivia. En el Perú, en la región de Cuzco se conservan las formas más arcáicas y menos castellanizadas del idioma, que llamaré 'Quichua Clásico'.

Los nativos de la región de Santiago no comprenden el Quichua tal como se habla en el norte del Ecuador, los que viven al sur de Mocoa parecen hablar una forma, más arcáica, intermediaria entre el Inga y el Quichua propiamente dicho que les permite hasta cierto punto la intercomprehensión con los indígenas del norte de la República vecina. El Inga de Santiago es probablemente el dialecto más diferenciado de la lengua de los conquistadores incáicos.

Esta segmentación del Inga se explica por el bilingüismo Quichua-Es. pañol ya bastante antiguo en la región del Putumayo, y presenta, en cierto modo, un caso típico de 'hibridación' de un sistema fonológico donde, desde el punto de vista diacrónico, los cambios fonemáticos de fonologización, desfonologización y transfonologización se explican completamente por la influencia del sistema fonológico de otra lengua.

Ese bilingüismo mucho más extenso en la época colonial, correspondía entonces a toda la zona ocupada por los Incas en la época de la conquista española y explica los numerosos quichuismos del español popular del Departamento de Nariño y del Sur del Departamento del Huila.

El contraste fonológico entre los fonemas del Inga ha sido determinado cada vez que haya sido posible por el método habitual de conmutaciones. Cuando ese método no se ha podido aplicar, se ha utilizado el método de oposiciones en contexto idéntico, o similar.

(3) Sin embargo, muchas formas han sido controladas por algunos informantes ocasionales oriundos de la misma región. 


\section{3.-Los Fonemas:}

El Inga utiliza 17 fonemas diferenciados: 14 consonantes y 3 vocales representados por los símbolos siguientes:

$$
\text { / p, t, ̌ , k, s, x, r, l, L, m, n, ñ, j, w, i, a, u / }
$$

CONSONANTES:

\section{CUADRO DE LOS FONEMAS (5)}

\section{Bilabiales Dentales Prepalatales Velares}

Oclusivas

(sordas) :

$$
\mathrm{p}
$$

$\mathbf{t}$

$\check{\mathrm{c}}$

Fricativas

$$
\text { (sordas): }
$$

Nasales

$$
\text { (sonoras) : }
$$

m

n

$\tilde{\mathbf{n}}$

Líquidas:

Laterales

(sonoras) :

Vibrante

(sonora) :

1

L

$\mathbf{r}$

SEMI-CONSO-

NANTES:

w

$$
\text { j }
$$

VOCALES:

Cerradas:

Abierta:

$\mathbf{u}$

a

(4) Indicaremos las notaciones fonológicas entre dos rayitas / /, según la costumbre norteamericana, para diferenciarla así claramente de las notaciones fonéticas que siempre aparecen entre paréntesis cuadrados [ ].

Usamos la notación de la Asociación Internacional de Fonética, excepto para la $l$ paladializada que notaremos /L/ y las semi-vocales [i] y [u] que distinguiremos de las vocales correspondientes por medio de bastardillas.

(5) Los Inganos usan también los sonidos siguientes en los préstamos del castellano [ $\mathrm{b} \mathrm{d} \mathrm{g} \mathrm{f}$ e o], pero estos fonemas, en español, alternan siempre

\begin{tabular}{|c|c|c|c|c|c|c|}
\hline $\begin{array}{l}{[\text { braso] }} \\
\text { [djos] } \\
\text { [alekre] } \\
\text { [flauta] }\end{array}$ & $\begin{array}{l}\mathbf{0} \\
\mathbf{0} \\
\mathbf{0} \\
0\end{array}$ & $\begin{array}{l}\text { [brasu] } \\
\text { [djus] } \\
\text { [alegri] } \\
\text { [flauxta }\end{array}$ & $\begin{array}{l}\mathbf{0} \\
\mathbf{0}\end{array}$ & $\begin{array}{l}\text { [praso] } \\
\text { [tjos] } \\
\text { [aligri] }\end{array}$ & $\begin{array}{l}= \\
= \\
= \\
=\end{array}$ & $\begin{array}{l}\text { /prasu/ } \\
\text { /tjus/ } \\
\text { /alikri/ } \\
\text { /wlawta/, }\end{array}$ \\
\hline
\end{tabular}
libremente con los fonemas Inga más cercanos y no representan sino variantes de aquellos:

Ejemplos:

$\begin{array}{lcc}{[\mathrm{b}]} & \text { variante de } & / \mathrm{p} / \\ {[\mathrm{d}]} & \# & / \mathrm{t} / \\ {[\mathrm{g}]} & \cdots & / \mathrm{k} / \\ {[\mathrm{e}]} & \cdots & / \mathrm{i} / \\ {[\mathrm{o}]} & \cdots & / \mathrm{u} / \\ {[\mathrm{f}]} & & / \mathrm{w} /\end{array}$


LISTA DE LOS FONEMAS CON EJEMPLOS ILUSTRATIVOS:

$\begin{array}{lll}\text { /p/ } & \text { /piki/ } & \text { 'nigua' } \\ \text { /t/ } & \text { /tuka/ } & \text { 'saliva' } \\ \text { /č/ } & \text { /čaki/ } & \text { 'pié' } \\ \text { /k/ } & \text { /kari/ } & \text { 'hombre' } \\ \text { /s/ } & \text { /sača/ } & \text { 'árbol' } \\ \text { /x/ } & \text { /xirumi/ } & \text { 'malo' } \\ \text { /m/ } & \text { /manka/ } & \text { 'hogar' } \\ \text { /n/ } & \text { /nina/ } & \text { 'candela' } \\ \text { /ñ } & \text { /ñawi/ } & \text { 'ojo' } \\ \text { /l/ } & \text { /lamuča/ } & \text { 'trapo' } \\ \text { /L/ } & \text { /LuLa/ } & \text { 'mentira' } \\ \text { /r/ } & \text { /rupa/ } & \text { 'calor' } \\ \text { /j/ } & \text { /jajkuj/ } & \text { 'entra!' } \\ \text { /w/ } & \text { /wanpra/ } & \text { 'niño' } \\ \text { /i/ } & \text { /inti/ } & \text { 'sol' } \\ \text { /a/ } & \text { /alpa/ } & \text { 'tierra' } \\ \text { /u/ } & \text { /učpa/ } & \text { 'ceniza' }\end{array}$

4.-Definición de los fonemas:

\section{LAS VOCALES}

Las Vocales del Inga se pueden clasificar según dos grados de abertura: abertura máxima y abertura mínima, y dos tipos de localización, sólo para el grado de abertura mínima: localización anterior y localización posterior.

Tiene, pues, un sistema vocálico triangular:

\section{Localización}

$\begin{array}{cc}\text { Anterior } & \text { Posterior } \\ \text { /i/ } & / \mathrm{u} /\end{array}$

Abertura:

Mínima:

Máxima:

Rasgos diferenciales:

Del punto de vista fonológico, estos fonemas vocálicos se pueden definir como sigue:

/i/ : fonema vocálico de abertura mínima y de localización anterior. 
/a/ : fonema vocálico de abertura máxima.

/u/ : fonema vocálico de abertura mínima y de localización posterior.

Estos tres fonemas vocálicos forman entre sí tres oposiciones con los rasgos distintivos siguientes:

Tipo de oposición

a / i . . . .... Abierta / cerrada

a / u . . . . . Abierta / cerrada

i / u ...... Anterior / posterior
Rasgo distintivo

Abertura

Abertura

Localización

\section{LAS CONSONANTES}

El Inga tiene en posición explosiva 14 fonemas consonánticos diferenciados que se clasifican por su modo de articulación en seis categorías: oclusivas, fricativas, nasales, laterales, vibrantes - las dos últimas forman, del punto de vista fonológico, la categoría de las líquidas- y semi-consonantes.

Los Seudo-Diptongos y su interpretación fonológica:

El Inga presenta las siguientes combinaciones de elementos vocálicos que pueden presentar una dificultad para el análisis:

$\begin{array}{llr}{[\mathrm{ai}]} & \mathrm{o} & {[\mathrm{aj}]} \\ {[\mathrm{au}]} & \mathrm{o} & {[\mathrm{aw}]} \\ {[\mathrm{ia}]} & 0 & {[\mathrm{ja}]} \\ {[\mathrm{ua}]} & 0 & {[\mathrm{wa}]} \\ {[\mathrm{ui}]} & 0 & {[\mathrm{wi}]} \\ {[\mathrm{iu}]} & \mathrm{o} & {[\mathrm{ju}]}\end{array}$

Los problemas que se plantean son los siguientes:

a) ¿Son estas agrupaciones de sonidos monofonemáticas o difonemáticas?

b) Si se interpretan como agrupaciones de fonemas diferentes, deben los elementos más cerrados de cada grupo $[i, \mathrm{j}, u, \mathrm{w}]$ interpretarse como variantes fonéticas de las vocales correspondientes /i, u/ o como semiconsonantes diferenciadas $/ \mathrm{j}, \mathrm{w} /$ ?

La primera pregunta es bastante fácil de contestar. Sabemos que "un grupo de sonidos sólo puede ser considerado como la realización de un fonema único si su duración no es más larga que la duración de otro 
fonema del idioma considerado" (6), los grupos vocálicos arriba mencionados son, pues, de duración mucho más larga que las vocales sencillas.

.Por otra parte, "cuando un elemento de una agrupación de sonidos, posiblemente monofonemática, no puede interpretarse como una variante combinatoria de algún fonema, la referida combinación debe valorarse como realización de un fonema particular" (7); los elementos [a i u] de las agrupaciones mencionadas siendo sin duda alguna realizaciones de los fonemas vocálicos, se excluye pues, la interpretación monofonemática de éstas.

En fin, motivos de orden puramente morfológico nos obligan también a negar la existencia de verdaderos diptongos en Inga. El imperativo de los verbos se forma al añadir el morfema [-i] a la forma radical, esta [i] forma con la vocal final de la forma radical un grupo vocálico, cuya pronunciación puede libremente variar desde dos vocales seguidas, en articulación lenta, hasta una vocal más una semi-consonante en la pronunciación rápida.

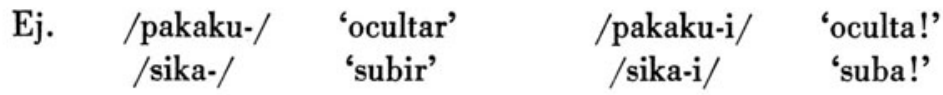

Como muchas formas radicales terminan en /-i/ tenemos numerosas agrupaciones del tipo [-ii] o [-ij]. Pero sabemos que "cuando dos sonidos emparentados entre sí acústicamente o muscularmente no aparecen nunca en contacto con unos mismos sonidos, deben considerarse como variantes combinatorias del mismo fonema" (8), aquí tenemos precisamente lo contrario, o sea la agrupación [ii] o [ij]; tenemos, pues, que considerar los dos elementos como distintos.

Esta misma regla nos permite contestar a la segunda pregunta también, por lo relacionado con el sonido $[i]$ o $[\mathrm{j}]$, pero no hemos encontrado ningún ejemplo del tipo [uw] o [wu] que nos permita diferenciar claramente el fonema /w/. Sin embargo, la estructura silábica del idioma nos permite aclarar este punto. En efecto, no parece posible hablar de agrupaciones de vocales ni de consonantes en la sílaba inga, con muy pocas excepciones, en préstamos del español. Todos los grupos consonán-

(6) Cf. N. Trubetzkoy. Anleitung zu phonologischen Beschreibungen, Brno. 1933, p. 13, Regeln VII.

(7) Cf. N. Trubetzkoy, Grundzüge der Phonologie, TCLP, 7, Regeln V|

(8) Cf. N. Trubetzkoy, Anleitung..., p. 9, Regeln III; y Grundzüge..., p. 44, Regeln III. 
ticos que se encuentran, se dividen claramente en dos sílabas distintas, -consonante implosiva + consonante explosiva - cada vez que se pida a un indígena de pronunciar de manera lenta una palabra que presenta un grupo de consonantes, corta el grupo como sigue:
/is-kaj/
'dos'
/pat-sa/
'cien'
/pič-ka/
'cinco'

La estructura silábica se puede pues sintetizar en la fórmula: (C) $\mathrm{V}(\mathrm{C})$ (9); es por consiguiente necesario interpretar los grupos vocálicos que nos ocupan como tipos CV o VC, así:

$\begin{array}{lll}\text { ai-ča } & = & \text { /ajča/ } \\ \text { či-ia } & = & \text { /čija/ } \\ \text { ju-ia } & = & \text { /juja/ }\end{array}$

Los sonidos $[i \mathrm{j} u \mathrm{w}]$ son, pues, siempre variantes combinatorias de los fonemas semi-consonantes $/ \mathrm{j} / \mathrm{y} / \mathrm{w} /$ respectivamente.

Interpretación fonológica de los sonidos: [tš] [nj] y [lj]:

El sonido africado [tš] está formado por dos elementos de los cuales uno [š] no existe como fonema en Inga, por consiguiente el grupo no puede interpretarse como la agrupación de dos elementos sino como un fonema sencillo $/ \check{c} /$, además, conmuta siempre en todas las posiciones con todos los demás fonemas consonánticos, y no queda dividido entre dos sílabas (10).

Un problema se plantea sin embargo cuando se trata de clasificarlo. Se puede considerarlo como una africada o como una oclusiva. La estructura general del sistema fonológico del idioma nos impone la interpretación oclusiva, no existe pues la africada /c/, cuando el sonido [ts] se encuentra en una palabra, los dos elementos pertenecen siempre a dos sílabas distintas. Nos parece entonces lógico considerar el fonema /č/ como una oclusiva prepalatal sorda.

Se debe notar que en Quichua peruano y boliviano, el fonema / $/ \check{c}$ es también oclusivo, en efecto, como en los otros puntos de articulación de las oclusivas existe tres fonemas distintos / $\check{\mathrm{c}} \check{\mathrm{c} c} \mathrm{c} \breve{\mathrm{c}} /$ / por las correlaciones de aspidación y de glotalización: / $\mathrm{p}$ p $\mathrm{p}^{\mathrm{P}}, \mathrm{t} \mathrm{t} \mathrm{t}^{\mathrm{P}}, \mathrm{k} \mathrm{k} \mathrm{k}^{2}, \mathrm{q} \mathrm{q} \mathrm{q}^{\mathrm{P}} /$. El Quichua clásico tiene pues cinco puntos de articulación de oclusivas: una serie bilabial, una dental, una prepalatal, una velar, y una posvelar.

(9) Hay algunas excepciones en préstamos del español y en pocas palabras indígenas:

$$
\mathrm{C}=\text { consonante } \quad \mathrm{V}=\text { vocal }
$$

(10) Cf. N. Trubetzkoy, Anleitung..., p. II, Regeln V. 
Los sonidos [nj] y [lj] también se pueden prestar a una doble interpretación, pero aquí otra vez la estructura del idioma, que no admite consonantes agrupadas, nos obliga a rechazar la interpretación difonemática a favor de la monofonemática / $\tilde{n} /$ y $/ \mathrm{L} /$

$\begin{array}{llll}\text { [njambi] } & \text { o } & \text { [ñambi] } & =/ \text { ñanpi/ } \\ \text { [nja-we] } & \text { o } & \text { [njawi] } & =/ \text { ñawi/ } \\ \text { [a-lyi-lyi] } & & & =/ \text { aLiLa/ } \\ \text { [nyitu } & \text { o } & \text { [nyito }] & =/ \text { ñitu/ del español 'nieto' }\end{array}$

Hay que notar en fin que tanto / $\tilde{n} /$ como $/ \mathrm{L} /$ se conmutan con todas las otras consonantes sencillas explosivas y que nunca se confunden con /n/ y /l/ respectivamente delante /i/:

/libra/ o /lipra/ 'libra' /Liki/ 'romper' /nina/ 'candela' /ñitu/ 'nieto'

Oposiciones y Rasgos Diferenciativos:

Los 14 fonemas consonánticos forman 91 oposiciones (11). Los tipos de oposición y los ragos pertinentes correspondientes son los siguientes:

a) oposición oral/nasal: p/m, t/n, č/n, (oposición privativa) Rasgo diferenciativo: nasalidad

b) oposición oclusiva/fricativa: $\mathrm{p} / \mathrm{w}, \mathrm{t} / \mathrm{s}, \check{\mathrm{c}} / \mathrm{j}, \mathrm{k} / \mathrm{x}$ (oposición privativa).

Rasgo diferenciativo: aproximación de los articuladores

c) oposición anterior/posterior: p/k, t/č, n/ñ, l/L, s/j, w/x

Rasgo diferenciativo: punto de articulación

d) oposición lateral/vibrante: $1 / \mathrm{r}$

Rasgo diferenciativo: simultaneidad o alternamiento de abertura o cerramiento.

e) oposición no liquida/liquida (lateral y vibrante) : r/l/L opuestos a todos los demás fonemas (oposición multilateral).

Rasgo diferenciativo: abertura y cerramiento.

(11) El número Q de oposiciones de un sistema fonológico de $n$ fonemas, se calcula con las fórmulas:

$$
\mathrm{Q}=\frac{\mathrm{n} 2-\mathrm{n}}{2} \quad \text { o } \quad \frac{\mathrm{n}(\mathrm{n}-1)}{2}
$$


Hay que notar que como el Quichua, el Inga no utiliza la oposición sorda/sonora (12).

Definición fonológica de los fonemas consonánticos:

/p/ : está đéfinido por las oposiciones: $\mathrm{p} / \mathrm{w}, \mathrm{p} / \mathrm{m}, \mathrm{p} / \mathrm{t}$, es pues oclusir. no-nasal y labial.

/t/ : está definido por las oposiciones siguientes: t/s, t/n, t/p,t t/c. es pues oclusivo, no-nasal y dental.

/c/ : está definido por las oposiciones siguientes: $\check{c} / \mathrm{j}, \check{c} / \tilde{\mathbf{n}}, \check{c} / \mathrm{t}, \check{c} / \mathbf{k}$. es pues oclusivo, no-nasal y prepalatal.

/k/ : está definido por las oposiciones siguientes: $\mathrm{k} / \mathrm{x}, \mathrm{k} / \mathrm{c}$, es pues oclusivo, velar.

$/ \mathrm{m} /$ : está definido por las oposiciones siguientes: $\mathrm{m} / \mathrm{p}, \mathrm{m} / \mathrm{n}$, es nasal, labial.

/n/ : está definido por las oposiciones siguientes: $n / t, n / m, n / \tilde{n}$, $\mathrm{n} / \mathrm{l}, \mathrm{n} / \mathrm{r}$,

es pues: nasal, dental, no-lateral, no-vibrante.

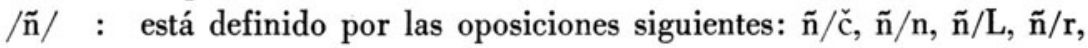
es pues nasal, prepalatal, no-lateral, no-vibrante.

/w/ : está definido por las oposiciones siguientes: w/p, w/m, m/s, es pues fricativo, no-nasal, labial.

/s/ : está definido por las oposiciones siguientes: $\mathrm{s} / \mathrm{t}, \mathrm{s} / \mathrm{n}, \mathrm{s} / \mathrm{w}, \mathrm{s} / \mathrm{j}$, es pues fricativo, no-nasal, y dental.

/j/ : está definido por las oposiciones siguientes: $\mathrm{j} / \check{\mathrm{c}}, \mathrm{j} / \tilde{\mathrm{n}}, \mathrm{j} / \mathrm{s}, \mathrm{j} / \mathrm{x}$, es pues fricativo, no-nasal, prepalatal.

/ $\mathrm{x}$ : está definido por las oposiciones siguientes: $\mathrm{x} / \mathrm{k}, \mathrm{x} / \mathrm{j}$, es pues fricativo, velar.

1/ : está definido por las oposiciones siguientes: l/n, l/r, l/L, l/ (todos los demás fonemas consonánticos), es pues no-nasal, no-vibrante, dental y lateral.

/L/ : está definido por las oposiciones siguientes: L/ñ, L/l, L/r, L/ (todos los demás fonemas consonánticos), es pues no-nasal, prepalatal, no-vibrante, lateral.

/r/ : está definido por las oposiciones siguientes: $r / n, r / \tilde{n}, r / l, r / L$, $\mathrm{r} /$ (todas las demás consonantes),

es pues no-nasal, no-lateral, anterior (puede variar entre la posición dental y prepalatal) y vibrante.

(12) č/j es una oposición de oclusión/fricción, sin que intervenga como rasgo diferenciativo la realización fonética sonora de $/ \mathrm{j} /$. 
En el diagrama siguiente se trató de representar de manera sintética los elementos que forman el consonantismo Inga y sus relaciones:

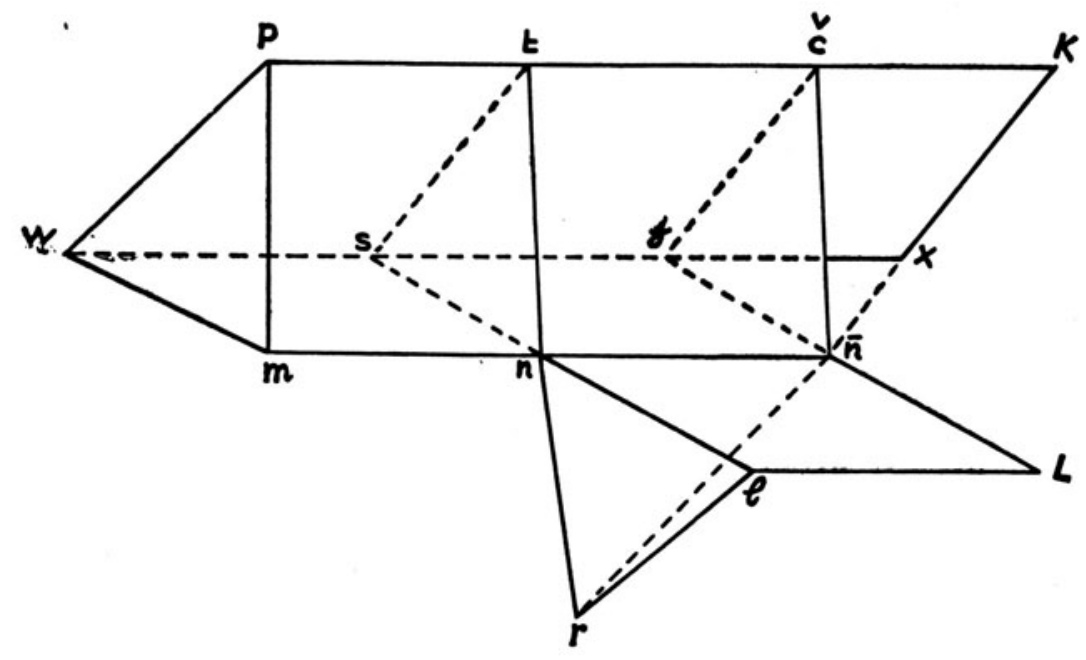

5.-Realización de los fonemas.

\section{LAS VOCALES}

El fonema /a/ se puede realizar entre la a anterior no labializada y la a posterior ligeramente labializada. Su articulación más común es la de a media con los labios neutros (13):

$\begin{array}{ll}\text { ačika } & \text { 'varios } \\ \text { ačka } & \text { 'largo, bastante' } \\ \text { alku } & \text { 'perro' } \\ \text { alpa } & \text { 'tierra' } \\ \text { ajča } & \text { 'carne' } \\ \text { amsa } & \text { 'oscuro' }\end{array}$

(13) Tiende a articularse palatal con los fonemas /t, s, č, j, n, ̃, l, L, r/; se articula velar o labializada después de los fonemas $/ \mathrm{p}, \mathrm{m}, \mathrm{w}, \mathrm{k}, \mathrm{x} /$. 


$\begin{array}{ll}\text { anaku } & \text { 'faldas' } \\ \text { anku } & \text { 'vena' } \\ \text { añanku } & \text { 'hormiga' } \\ \text { asawači } & \text { 'pulsera' } \\ \text { asLita } & \text { 'poco' } \\ \text { aswa } & \text { 'chicha' } \\ \text { atun } & \text { 'alto, grande' } \\ \text { awa } & \text { 'arriba' } \\ \text { axča } & \text { 'pelo' }\end{array}$

El fonema /i/ se realiza entre la $i$ cerrada y la e cerrada, sin que el contexto fónico presente explicación alguna en muchos casos (14) :

$\begin{array}{ll}\text { kiLa } & \text { 'luna' } \\ \text { kimsa } & \text { 'tres' } \\ \text { kiLu } & \text { 'amarillo' } \\ \text { mikwi } & \text { 'comida' } \\ \text { misitu } & \text { 'gato' } \\ \text { miski } & \text { 'sabroso' } \\ \text { xiromi } & \text { 'mal' }\end{array}$

El fonema /u/ se realiza entre la $u$ cerrada labializada y la o cerrada labializada (15) :

$\begin{array}{ll}\text { uču } & \text { 'ají' } \\ \text { učpa } & \text { 'ceniza' } \\ \text { učuLa } & \text { 'pequeño' } \\ \text { ukupi } & \text { 'dentro' } \\ \text { uma } & \text { 'cabeza' } \\ \text { umutu } & \text { 'corto' } \\ \text { unkux } & \text { 'enfermo' } \\ \text { upa } & \text { 'tonto' } \\ \text { ura } & \text { 'abajo' } \\ \text { usa } & \text { 'piojo' } \\ \text { uxsa } & \text { 'hierba' } \\ \text { čupa } & \text { 'rabo' } \\ \text { čuču } & \text { 'seno' }\end{array}$

(14) Tiende a articularse [i] después de consonantes palatales /t, s, č, i, $\mathrm{L}, \mathrm{n}, \tilde{\mathrm{n}} / \mathrm{y}$ de $/ \mathrm{m}, \mathrm{x} /$. Se pronuncia indiferentemente [i] o [e] cerrada después de las demás consonantes.

(15) Se pronuncia [u] después de las consonantes labiales y velares, e indiferentemente entre $[\mathrm{u}]$ y [o] cerrada después de las demás consonantes. 


$\begin{array}{ll}\text { čunka } & \text { 'diez' } \\ \text { kuču } & \text { 'orilla' } \\ \text { kulki } & \text { 'plata' } \\ \text { Lunči } & \text { 'pared' } \\ \text { muču } & \text { 'beso' } \\ \text { musu } & \text { 'nuevo' } \\ \text { puka } & \text { 'rojo' }\end{array}$

Nota: Las tres vocales se nasalizan cada vez que se encuentran en sílaba cerrada por /-n/ seguida de /k-/ en la sílaba siguiente:

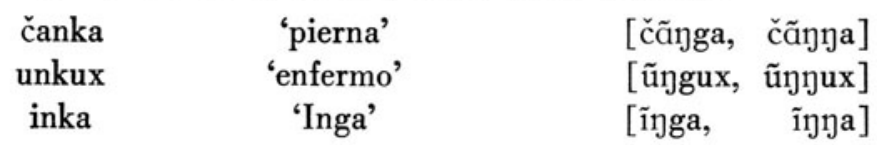

\section{LAS CONSONANTES}

/p/ : se articula como una oclusiva bilabial sorda en todas las posiciones, excepto después de /n/ donde se sonoriza en [b], la /n/ pronunciándose $[\mathrm{m}]$ por asimilación de punto de articulación (16):

[p]

$\begin{array}{ll}\text { patsa } & \text { 'cien' } \\ \text { paj } & \text { 'él' } \\ \text { kajpi } & \text { 'aqui' } \\ \text { rupaku } & \text { 'llama' } \\ \text { kalpa- } & \text { 'correr' } \\ \text { wanpra } & \text { 'niño' } \\ \text { anpi } & \text { 'curar' } \\ \text { čanpa } & \text { 'zanja' } \\ \text { čunpi } & \text { 'faja' }\end{array}$

[wambra]

[b]

[ambi]

[čamba]

[čumbi]

/t/ : se articula como una oclusiva dental sorda en todas las posiciones, excepto después de /n/ donde se sonoriza en [d], la /n/ se pronuncia siempre como una oclusiva nasal dental:

$[\mathrm{t}]$

$\begin{array}{ll}\text { tajta } & \text { 'padre' } \\ \text { tuta } & \text { 'noche' } \\ \text { tanpu } & \text { 'rancho' } \\ \text { katanka } & \text { 'cobija' } \\ \text { pitaka } & \text { 'quién?' }\end{array}$

(16) En esta posición, la oposición $\mathrm{n} / \mathrm{m}$ se neutraliza; pero se conserva siempre delante de $/ \mathrm{t} /$, ejemplos:

$$
\text { y } / \text { mamta/ }=[\text { manda }] \quad \text { 'tender' },
$$




\begin{tabular}{|c|c|c|c|}
\hline \multirow[t]{5}{*}{ [d] } & manta & 'tender' & [manda] \\
\hline & ranti- & 'comprar' & [randi-] \\
\hline & aLimanta & 'despacio' & [aLimanda] \\
\hline & čisimanta & 'anoche' & [čisimanda] \\
\hline & inti & 'sol' & [indi] \\
\hline
\end{tabular}

/k/ : se articula como una oclusiva velar sorda en todas las posiciones después de $/ \mathrm{n} /$ donde se pronuncia [g] y a veces se nasaliza en oclusiva nasal velar $[\mathrm{y}]$; la $/ \mathrm{n} /$ anterior se velariza también por asimilación del punto de articulación y el grupo geminado así formado se simplifica en la pronunciación rápida. En resumen, el grupo /-nk-/ se puede pronunciar [-ng-], [-ng-], [-1 y-], o [-n-]. La vocal que viene antes de la $/ \mathrm{n} /$ se nasaliza también por asimilación regresiva:

\begin{tabular}{|c|c|c|c|}
\hline \multirow[t]{5}{*}[\mathrm{k}]{} & kari & \multicolumn{2}{|l|}{ 'hombre' } \\
\hline & kuri & 'oro' & \\
\hline & kam & 'tú' & \\
\hline & piki & 'nigua' & \\
\hline & waka- & 'llorar’ & \\
\hline [g] & čanka & 'pierna' & [čãnga, čãnga, čãyıa, čãna] \\
\hline o & añanku & 'hormiga' & 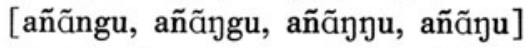 \\
\hline$[\mathrm{y}]$ & unkux & 'enfermo' & 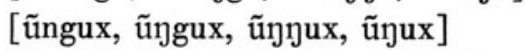 \\
\hline & inkanu & 'Ingano' & [ĩnganu, ĩnganu, ĩ̄yanu, ĩ̄anu ] \\
\hline & čunka & 'diez' & [čũnga, čũnga, čũña, čũ̃a] \\
\hline
\end{tabular}

/č/ : se realiza entre una africada mediopalatal formada de un elemento oclusivo alveolar y de un elemento fricativo mediopalatal, ambos sordos, y una oclusiva palatalizada de articulación media. Es siempre sordo:

$\begin{array}{ll}\text { čanka } & \text { 'pierna' } \\ \text { čunka } & \text { 'diez' } \\ \text { čaki } & \text { 'pié' } \\ \text { čara } & \text { 'todavía' } \\ \text { či } & \text { 'aquel' } \\ \text { čisi } & \text { 'tarde' } \\ \text { čuspi } & \text { 'mosca' } \\ \text { ajča } & \text { 'carne' } \\ \text { asawači } & \text { 'pulsera' } \\ \text { kači } & \text { 'sal' }\end{array}$

/s/ : se realiza como una fricativa sorda dental o alveolar: 


$\begin{array}{ll}\text { sača } & \text { 'árbol, monte' } \\ \text { sačuku } & \text { 'montaña' } \\ \text { saparu } & \text { 'canasta' } \\ \text { sara } & \text { 'maíz' } \\ \text { siLu } & \text { 'uña' } \\ \text { simi } & \text { 'boca' } \\ \text { sinka } & \text { 'nariz' } \\ \text { sinči } & \text { 'médico' } \\ \text { suma } & \text { 'bello' } \\ \text { suxu } & \text { 'uno' } \\ \text { aswa } & \text { 'chicha' } \\ \text { časiska } & \text { 'seco' } \\ \text { čisi } & \text { 'tarde' } \\ \text { čusku } & \text { 'cuatro' } \\ \text { iskaj } & \text { 'dos' }\end{array}$

/x/ : se realiza como una fricativa velar sorda:

$\begin{array}{ll}\text { Xakanamixoj } & \text { (Apellido) } \\ \text { xirumi } & \text { 'estar mal' } \\ \text { xuntači } & \text { 'llenar' } \\ \text { axča } & \text { 'pelo' } \\ \text { čuxLu } & \text { 'mazorca' } \\ \text { ñaxča } & \text { 'peine' } \\ \text { uxsa } & \text { 'hierba' } \\ \text { suxpunča } & \text { 'pasado mañana' } \\ \text { pusax } & \text { 'ocho' }\end{array}$

/r/ : se articula como una vibrante apical sonora en todas las posiciones:

$\begin{array}{ll}\text { ri- } & \text { 'ir' } \\ \text { riksi- } & \text { 'conocer' } \\ \text { rima- } & \text { 'hablar' } \\ \text { rinri } & \text { 'oreja' } \\ \text { ruku } & \text { 'viejo, anciano' } \\ \text { rukusača } & \text { 'selva' } \\ \text { rumi } & \text { 'piedra' } \\ \text { rumu } & \text { 'yuka' } \\ \text { rupa } & \text { 'calor' } \\ \text { rupariku } & \text { 'fuego, llama' } \\ \text { ari } & \text { 'asi'' }\end{array}$




$\begin{array}{ll}\text { arma- } & \text { 'bañar' } \\ \text { atari- } & \text { 'levantarse' } \\ \text { čuru } & \text { 'caracol' } \\ \text { kara } & \text { 'piel' } \\ \text { kari } & \text { 'hombre' } \\ \text { kuru } & \text { 'gusano' } \\ \text { kunkur } & \text { 'rodilla' } \\ \text { kurki } & \text { 'plata' }\end{array}$

1/ : se realiza como una lateral alveolar sonora en todas las posiciones:

$\begin{array}{ll}\text { lamuča } & \text { 'trapo' } \\ \text { linpia- } & \text { 'limpiar' } \\ \text { alku } & \text { 'perro' } \\ \text { alpa } & \text { 'tierra' } \\ \text { ačala taita } & \text { 'abuelo' } \\ \text { čalwa } & \text { 'pez' } \\ \text { činkwal } & \text { 'ciego; bizco' } \\ \text { kalpa- } & \text { 'correr' } \\ \text { kulki } & \text { 'plata' } \\ \text { jali- } & \text { 'pasar' }\end{array}$

/L/ : se realiza como una lateral mediopalatal sonora en todas las posiciones:

$\begin{array}{ll}\text { Laki } & \begin{array}{l}\text { 'pena' } \\ \text { Lakispa }\end{array} \\ \text { Lamka- } & \text { 'tristeza' } \\ \text { Lasa } & \text { 'pesado' } \\ \text { Liki } & \text { 'romper' } \\ \text { LuLa } & \text { 'mentira' } \\ \text { LuLu } & \text { 'tierno' } \\ \text { Linči } & \text { 'pared' } \\ \text { Lusi- } & \text { 'salir' } \\ \text { aLanka } & \text { 'quizás' } \\ \text { aLiLa } & \text { 'bien' } \\ \text { aLiči- } & \text { 'acomodar' } \\ \text { aLimanta } & \text { 'despacio' } \\ \text { asLita } & \text { 'poco' } \\ \text { čiLapi } & \text { 'allá' } \\ \text { čuxLu } & \text { 'mazorca' } \\ \text { kaLu } & \text { 'labio' }\end{array}$


/m/ : se realiza como una consonante oclusiva nasal bilabial sonora en todas las posiciones:

$\begin{array}{ll}\text { mačasi } & \text { 'receloso, vergonzoso' } \\ \text { majma } & \text { 'dónde' } \\ \text { maki } & \text { 'mano, antebrazo' } \\ \text { mama ninku } & \text { 'madrasta' } \\ \text { mana } & \text { 'no' } \\ \text { manka } & \text { 'hogar' } \\ \text { manima } & \text { 'nada' } \\ \text { mapa } & \text { 'sucio' } \\ \text { mikuj } & \text { 'comida' } \\ \text { ministiwa- } & \text { 'necesitar' } \\ \text { misitu } & \text { 'gato' } \\ \text { miski } & \text { 'sabroso' } \\ \text { muču } & \text { 'beso' } \\ \text { muna- } & \text { 'amar' } \\ \text { musu } & \text { 'nuevo' } \\ \text { amsa } & \text { 'oscuro, de noche' } \\ \text { arma- } & \text { 'bañar' } \\ \text { čima } & \text { 'allá' } \\ \text { kam } & \text { 'tú' } \\ \text { jamta } & \text { 'leña' }\end{array}$

/n/ : se pronuncia como una oclusiva nasal sonora dental en todas las posiciones excepto en el interior de palabras en contacto con una bilabial /p/ donde se articula bilabial por asimilación del punto de articulación y en la misma posición delante la gutural $/ \mathrm{k}$ / donde se pronuncia nasal sonora velar también por asimilación del punto de articulación:

$\begin{array}{lll}\text { [n] } & \text { nina } & \text { 'candela' } \\ \text { ninakaspi } & \text { 'tizón' } \\ \text { nuka } & \text { 'yo' } \\ \text { nukanči } & \text { 'nosotros' } \\ \text { ajni- } & \text { 'contestar' } \\ & \text { aLimanta } & \text { 'despacio' } \\ & \text { aLinči } & \text { 'componer' } \\ & \text { anaku } & \text { 'faldas' } \\ & \text { ančuri- } & \text { 'quitarse' } \\ & \text { atun } & \text { 'alto, grande' } \\ & \text { inti } & \text { 'sol' } \\ & \text { čunpi } & \text { 'faja' }\end{array}$




\begin{tabular}{|c|c|c|c|}
\hline \multirow{8}{*}[y]{} & čanpa & 'zanja' & [čamba] \\
\hline & anbi- & 'curar' & [ambi-] \\
\hline & inka & 'Inga' & [ĩnga, ĩnga, ĩña, ĩna $]$ \\
\hline & aLanka & 'quizás' & $\begin{array}{l}\text { [aLãnga, aLãyga, aLãyıa, } \\
\text { aLãya ] }\end{array}$ \\
\hline & añanku & 'hormiga' & 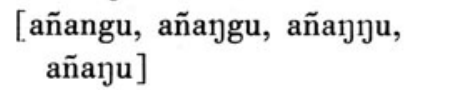 \\
\hline & čanka & 'pierna' & [čãnga, čãnga, čãña, čãya] \\
\hline & činkwal & 'ciego' & $\begin{array}{l}\text { [čĩngwal, čĩngwal, čĩnywal, } \\
\text { čĩnwal] }\end{array}$ \\
\hline & čunka & 'diez' & [čũnga, čũ̃ga, čũỹa, čũya] \\
\hline
\end{tabular}

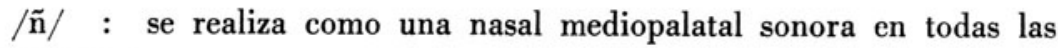
posiciones:

$\begin{array}{ll}\text { ñanpi } & \text { 'camino' } \\ \text { ñañu } & \text { 'delgado' } \\ \text { ñawi } & \text { 'ojo' } \\ \text { ñaxča } & \text { 'peine' } \\ \text { nuxpa } & \text { 'primero' } \\ \text { añanku } & \text { 'hormiga' } \\ \text { mañačiwa } & \text { 'prestar' }\end{array}$

/j/ : se realiza en la mayoría de los casos como una semi-consonante palatal no labializada en posición inicial de sílaba y como una semi-vocal $[i]$ en posición final de sílaba. Esta distinción articulatoria se debe probablemente a la influencia de la pronunciación española que presenta precisamente la misma distinción de carácter puramente fonético.

$\begin{array}{cll}\text { [j] } & \text { jačači- } & \text { 'enseñar' } \\ \text { jaku } & \text { 'agua' } \\ & \text { jali- } & \text { 'pasar' } \\ & \text { jamta } & \text { 'leña' } \\ & \text { jana } & \text { 'negro' } \\ & \text { jura } & \text { 'blanco' } \\ & \text { juja- } & \text { 'pensar' } \\ & \text { ajča } & \text { 'carne' } \\ & \text { jajkuj } & \text { 'entre' } \\ & \text { jačajku- } & \text { 'aprender' } \\ & \text { ajni- } & \text { 'contestar' } \\ & \text { ajsa- } & \text { 'sacar' } \\ & \text { čajpi } & \text { 'allá' }\end{array}$


/w/ : se realiza generalmente como una semiconsonante velar labializada en la inicial de palabra y como semi-vocal $[u]$ en posición implosiva (17). Como en el caso de / $\mathrm{j} /$ esta distinción articulatoria viene con toda seguridad de la influencia del español:

$\begin{array}{cll}\text { [w- }] & \text { waka- } & \text { 'llorar' } \\ & \text { wakuči- } & \text { 'colgar' } \\ & \text { wamra } & \text { 'niño' } \\ & \text { warmi } & \text { 'mujer' } \\ & \text { wasi } & \text { 'casa' } \\ & \text { wata } & \text { 'año' } \\ & \text { wiki } & \text { 'lágrima' } \\ & \text { wičaj } & \text { 'cuesta' } \\ & \text { wira } & \text { 'grasa' } \\ & \text { wiru } & \text { 'caña' } \\ & \text { wisa } & \text { 'estómago' } \\ & \text { čawxu } & \text { 'mitad' }\end{array}$

\section{6.-Tratamiento Prosódico:}

El acento tónico cae generalmente en la penúltima sílaba de las unidades significativas, pero nunca sirve para diferenciarlas:

$$
\begin{array}{lcll}
\text { ej.-—: } & \text { upjan'kapa } & = & \text { (para) beber } \\
\text { 'nuka } & \text { ‘jaku up'jasa } & = & \text { voy a beber agua } \\
\text { 'nuka ‘jaku mi upja'kumi } & = & \text { estoy bebiendo agua } \\
\text { 'kajna } & \text { 'jaku mi upjar'kani } & = & \text { ayer he bebido agua }
\end{array}
$$

Existe, por otra parte, un acento enfático combinado con un tono ascendiente de la vocal donde cae, que generalmente se coloca sobre la sílaba radical de la unidad significativa que se debe poner en relieve, y que provoca al mismo tiempo un alargamiento de la vocal:

ej.-: čisi'manta waka'čiska $=$ guardado de anoche čisi'manta wa'ka:čiska = guardado de anoche

'nuka a'miku kawar'kami 'pajpa wan'prata 'nuka pa'nipa xar'dimpi $=$ mi amigo vió a su hijo en el jardín de mi hermano.

'nuka a'miku kawar'kami 'pajpa wan'prata 'nuka 'pa:nipa xar'dimpi $=$ mi amigo vió a su hijo en el jardín de mi hermano!

(17) Tenemos muy pocos ejemplos de $[u]$ implosivo y alternan generalmente con -ux o sencillamente $-\mathrm{x}$ en casi todos los casos. Parece pues que hay neutralización de la oposición w/x en esta posición. 
in'kanu'kuna mi'kanči

in'ka:nukuna mi'kanči
$=$ somos Inganos

$=$ los Inganos, somos nosotros!

En resumen, el Inga es un idioma prosódicamente neutro. Su acento fonético en la penúltima sílaba de la unidad significativa tiende a desarrollar un acento secundario binario en cada dos sílabas anteriores, cuando la palabra tiene más de tres sílabas en total. Tiene, además, un acento de tipo afectivo o estilístico extrafonológico que se localiza sobre la sílaba radical de la unidad significativa y produce una alza de tono y un alargamiento de la vocal.

\section{7.-Estructura de la Sílaba:}

Cualquier palabra Inga consiste en una o varias sílabas. Cada sílaba tiene un punto acústicamente más alto por su sonoridad que corresponde siempre a un fonema vocálico. Ese fonema vocálico puede formar por sí solo la sílaba, pero puede también estar precedido o seguido, o precedido y seguido por un fonema consonántico, excepto en algunas palabras de origen español, donde se encuentran agrupaciones de fonemas consonánticos en la misma sílaba y en algunas palabras indígenas que admiten las agrupaciones $\mathrm{Cj}$ y $\mathrm{Cw}$, iniciales de sílaba. En palabras genuinamente indígenas, los grupos consonánticos sólo se encuentran interiores de palabra, y, en este caso la primera consonante pertenece a la sílaba anterior (consonante implosiva) y la segunda a la sílaba siguiente (consonante explosiva).

En resumen, la sílaba Inga puede presentar las estructuras siguientes: ( $\mathrm{V}=$ fonema vocálico $; \mathrm{C}=$ fonema consonántico $)$.

\begin{tabular}{ccc} 
& \multicolumn{1}{c}{ V } & \multicolumn{1}{c}{ CV (18) } \\
& & \multicolumn{1}{c}{ CVC } \\
$-\mathrm{V} \quad:$ & & Ejemplos \\
& ačika & 'varios' \\
& ima & 'qué, cualquier cosa' \\
& ura & 'abajo' \\
& upa & 'tonto' \\
umutu & 'corto'
\end{tabular}

(18) Como ya lo notamos, los rarísimos tipos CCV ocurren en palabras de origen español o con las agrupaciones $\mathrm{Cj}$ y $\mathrm{Cw}$. 


\begin{tabular}{|c|c|c|}
\hline & ačka & 'largo; bastante' \\
\hline & alku & 'perro' \\
\hline & alpa & 'tierra' \\
\hline & anpi- & 'curar' \\
\hline & amsa- & 'oscuro' \\
\hline & anku & 'raíz, vena' \\
\hline & arma- & 'bañar' \\
\hline & asLita & 'poco' \\
\hline & axča & 'pelo' \\
\hline & inti & 'sol' \\
\hline & inka & 'inga' \\
\hline & iskai & 'dos' \\
\hline & učpa & 'ceniza' \\
\hline & unkux & 'enfermo' \\
\hline & urma- & 'caer' \\
\hline & uxsa & 'hierba' \\
\hline CV & : & \\
\hline & čaki & 'pie' \\
\hline & kači & 'sal' \\
\hline & muna- & 'querer' \\
\hline & nina & 'candela' \\
\hline & piki & 'nigua' \\
\hline & ruku & 'viejo, anciano' \\
\hline & sača & ‘árbol’ \\
\hline & tuka & 'saliva' \\
\hline & xirumi & 'estar mal' \\
\hline CVC & : & \\
\hline & čanpa & 'sanja' \\
\hline & čanka & 'pierna' \\
\hline & čapsi- & 'sacudir' \\
\hline & časki- & 'recibir' \\
\hline & činkwal & 'ciego’ \\
\hline & čunpi & 'faja' \\
\hline & čunka & 'diez' \\
\hline & čusku & 'cuatro' \\
\hline & čuxu & 'mazorca' \\
\hline & kalpa- & 'correr' \\
\hline & kam & 'tú' \\
\hline & kančis & 'siete' \\
\hline & kaspi & 'palo' \\
\hline
\end{tabular}




$\begin{array}{ll}\text { kimsa } & \text { 'tres' } \\ \text { kinča } & \text { 'cerco' } \\ \text { kurki } & \text { 'plata' } \\ \text { amka- } & \text { 'tocar' } \\ \text { miski } & \text { 'sabroso' } \\ \text { ñaxca } & \text { 'peine' } \\ \text { punča } & \text { 'claro' } \\ \text { punku } & \text { 'puerta' } \\ \text { sinči } & \text { 'médico' } \\ \text { tipsi- } & \text { 'pellizar' }\end{array}$

8.-Distribución de los fonemas:

Consonantes sencillas

a) Iniciales:

Todos los fonemas consonánticos pueden ocurrir en posición inicial de sílaba delante de todas las vocales.

$\begin{array}{lll}\text { /p/ } & \text { paj } & \text { 'él' } \\ \text { pača } & \text { 'faldas' } \\ \text { panpa } & \text { 'valle' } \\ \text { pani } & \text { 'hermano' } \\ \text { pita } & \text { 'quien' } \\ \text { piki } & \text { 'nigua' } \\ \text { pička } & \text { 'cinco' } \\ \text { punča } & \text { 'día' } \\ \text { pukusku } & \text { 'maduro' } \\ & \text { pupu } & \text { 'ombligo' } \\ & \text { tajta } & \text { 'padre' } \\ \text { tanpu } & \text { 'rancho' } \\ & \text { tanta } & \text { 'pan' } \\ \text { tjami } & \text { 'hay' } \\ \text { tinpuči } & \text { 'hervir' } \\ \text { tipsi- } & \text { 'pellizar' } \\ \text { tuka } / & \text { 'saliva' } \\ & \text { tuLu } & \text { 'hueso' } \\ & \text { tuta } & \text { 'noche' } \\ & \text { kača- } & \text { 'enviar' } \\ & \text { kači } & \text { 'sal' } \\ \text { kaspi } & \text { 'palo' }\end{array}$




\begin{tabular}{|c|c|c|}
\hline & kiLa & 'luna' \\
\hline & kimsa & 'tres' \\
\hline & kiLu & 'amarillo' \\
\hline & kuri & 'oro’' \\
\hline & kuči & 'puerco' \\
\hline & kuču & 'orilla' \\
\hline$/ \check{c} /$ & čaki & 'pié' \\
\hline & čanka & 'pierna' \\
\hline & čara & 'todavía' \\
\hline & či & 'aquél' \\
\hline & čima & 'allá' \\
\hline & čisi & 'tarde' \\
\hline & čupa & 'rabo' \\
\hline & čuču & 'seno' \\
\hline & čusa & ‘vacío' \\
\hline /s/ & sača & 'árbol, monte' \\
\hline & samai & 'respiración' \\
\hline & sara & 'maíz' \\
\hline & siLu & 'luna' \\
\hline & $\operatorname{simi}$ & 'boca' \\
\hline & sinka & 'nariz' \\
\hline & suma & 'bello' \\
\hline & sutipa & 'cierto' \\
\hline & sunku & 'hígado' \\
\hline$/ \mathrm{x} /$ & xakanamixui & (Apellido) \\
\hline & xirumi & 'estar mal' \\
\hline & xuntači- & 'llenar' \\
\hline$/ \mathrm{m} /$ & maki & 'mano' \\
\hline & mapa & 'sucio' \\
\hline & mana & 'no' \\
\hline & mikui & 'comida' \\
\hline & misitu & 'gato' \\
\hline & miski & 'sabroso' \\
\hline & muča- & 'besar' \\
\hline & muna- & 'amar' \\
\hline & musu & 'nuevo' \\
\hline$/ \mathrm{n} /$ & nina & 'candela' \\
\hline & anaku & 'faldas' \\
\hline & nuka & 'yo’' \\
\hline$/ \tilde{\mathbf{n}} /$ & ñanpi & 'camino' \\
\hline
\end{tabular}




$\begin{array}{lll} & \text { ñanu } & \text { 'delgado' } \\ \text { nuxpa } & \text { 'primero' } \\ \text { lipra } & \text { 'libra' } \\ \text { lamuča } & \text { 'trapo' } \\ \text { ačalataita } & \text { 'abuelo' } \\ \text { čalwa } & \text { 'pez' } \\ \text { Lakispa } & \text { 'tristeza' } \\ \text { Lamka- } & \text { 'tocar' } \\ \text { Lasa } & \text { 'pesado' } \\ \text { Liki- } & \text { 'romper' } \\ \text { LuLa } & \text { 'mentira' } \\ \text { LuLu } & \text { 'tierno' } \\ \text { Lunči } & \text { 'pared' } \\ \text { ranti- } & \text { 'comprar' } \\ \text { ri- } & \text { 'ir' } \\ \text { riksi- } & \text { 'comer' } \\ \text { rima- } & \text { 'hablar' } \\ \text { ruku } & \text { 'anciano' } \\ & \text { rumi } & \text { 'piedra' } \\ \text { rumu } & \text { 'yuca' }\end{array}$

b) Mediales:

Ningún fonema consonántico puede ocupar una posición medial en la sílaba, esta localización siendo reservada a un fonema vocálico por su mayor sonoridad. Los fonemas consonánticos en posición medial de palabra o sea entre vocales pertenecen siempre a la sílaba siguiente:

$$
\text { mu.ča- 'besar' }
$$

nunca se puede cortar la palabra así: muč.a

Los fonemas consonánticos en posiciones semejantes son en realidad iniciales de sílaba.

c) Finales:

Sólo pueden ser finales de sílaba los fonemas siguientes:

$$
\text { / } \mathrm{p}, \mathrm{t}, \mathrm{k}, \check{\mathrm{c}}, \mathrm{s}, \mathrm{x}, \mathrm{m}, \mathrm{n}, \mathrm{l}, \mathrm{r}, \mathrm{j} /
$$

(19) Se debe notar que en algunos casos /-r/ y /-x/ implosivos delante de $/ \mathrm{k} /$ explosivo se neutralizan. 


$\begin{array}{lll}\text { /-p/ } & \text { čap-si- } & \text { 'sacudir' } \\ \text { /-t/ } & \text { pat.sa } & \text { 'cien' } \\ \text { /-k/ } & \text { rik.si- } & \text { 'conocer' } \\ \text { /-č/ } & \text { ač.ka } & \text { 'largo, bastante' } \\ \text { /-s/ } & \text { is.kun } & \text { 'nueve' } \\ \text { /-x/ } & \text { ax.ča } & \text { 'pelo' } \\ \text { /-m/ } & \text { wam.ra } & \text { 'niño' } \\ \text { /-n/ } & \text { am.sa } & \text { 'oscuro' } \\ /-\mathrm{l} / & \text { kul.ki } & \text { 'plata' } \\ \text { /-r/ } & \text { mar.kas.ka } & \text { 'bautismo' } \\ \text { /-j/ } & \text { rij } & \text { 'entra!' }\end{array}$

Como se puede notar, hay neutralización de los fonmeas $/ \mathrm{L} / \mathrm{y} / \tilde{\mathrm{n}} /$ $\operatorname{con} / \mathrm{l} / \mathrm{y} / \mathrm{n} /, \mathrm{y} / \mathrm{w} / \operatorname{con} / \mathrm{x} /$ respectivamente (20), en cualquier posición implosiva. Cuando estos fonemas no ocurren en final de palabra sino en final de sílaba, se presentan algunas otras neutralizaciones por influencia de las consonantes iniciales de la segunda sílaba, como por ejemplo: /n/ y /m/ delante /p-/ (21).

\section{Consonantes agrupadas}

En Inga no ocurren nunca agrupaciones de consonantes en la sílaba (22). Los grupos consonánticos interiores de palabra vienen siempre del encuentro de una consonante implosiva con una consonante explosiva que pertenecen a dos sílabas distintas. Como ciertas consonantes explosivas producen por asimilación la neutralización de ciertos fonemas en posición implosiva es necesario determinar con exactitud cuáles son las combinaciones posibles entre los once fonemas consonánticos que se pueden encontrar en posición final de sílaba y los catorce fonemas consonánticos que pueden encontrarse en posición inicial de sílaba:

(20) El español 'flauta' da en Inga [flawta, flawxta, flaxta], el tipo fonológico es /wlaxta/ o /wlawta/.

(21) Sólo la etimología puede distinguir / kampi/ 'de ti, tuyo' de /ñanpi/ 'camino', donde los grupos /-mp-/ y /-np-/ se pronuncian en ambos ca$\operatorname{sos}[\mathrm{mb}]$.

(22) Con las excepciones anotadas anteriormente. 


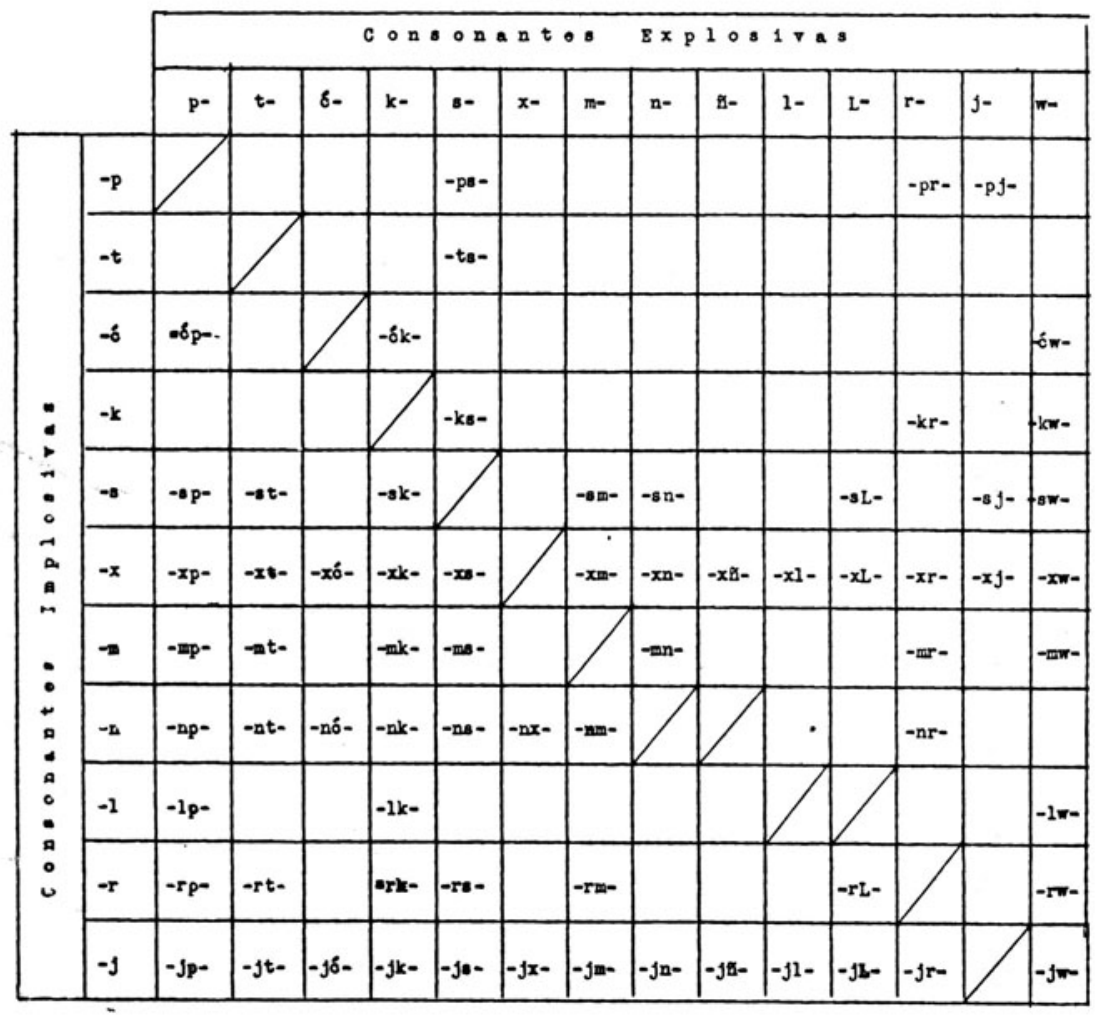

De las 152 agrupaciones teóricamente posibles hay que eliminar en primer lugar los grupos de consonantes similares, pues, el Inga no admite consonantes geminadas. En segündo lugar hay que suprimir también los grupos /-nñ-/ y /-lL-/ porque /n/ y /l/ implosivos representan también /-ñ / y /L/ neutralizados en esta posición y los grupos anteriormente citados son en realidad consonantes geminadas reducidas en la pronunciación.

Entre los 141 grupos restantes, hemos podido verificar la existencia de sólo 71 , o sea aproximadamente $50 \%$ de las posibilidades, pero naturalmente un conocimiento más extenso del vocabulario Inga podrá pro- 
bablemente aumentar sensiblemente el número de combinaciones realizadas.

Hé aquí una lista de ejemplos típicos de las agrupaciones encontradas:

\begin{tabular}{|c|c|c|}
\hline$/-p s-/$ & tipsi- & 'pellizar' \\
\hline /-ts-/ & patsa & 'cien' \\
\hline /-ks-/ & riksi- & 'conocer' \\
\hline /-с̌р-/ & učpa & 'ceniza' \\
\hline /-čk-/ & ačka & 'bastante' \\
\hline /-sp-/ & kaspi & 'madera' \\
\hline /-st-/ & asta- & 'acarrear' \\
\hline /-sk-/ & markaska & 'bautismo' \\
\hline /-sm-/ & ismuska & 'podrido' \\
\hline /-sn-/ & kusni & 'humo' \\
\hline /-xp-/ & ñuxpa & 'primero' \\
\hline /-xt-/ & suxta & 'seis' \\
\hline /-xk-/ & waxki & 'hermano' \\
\hline |-xč- $/$ & axča & 'pelo' \\
\hline /-xs-/ & kawarixsamanunkapa & 'para venir a comparecer' \\
\hline /-xm-/ & wañuxmanta & 'se murió' \\
\hline /-xL-/ & čuxlu & 'mazorca' \\
\hline /-xr-/ & waxra & 'ganado' \\
\hline /-mp-/ & kampa & 'tuyo' \\
\hline /-mt-| & kamta & 'contigo' \\
\hline /-mk-/ & kamkuna & 'vosotros' \\
\hline /-ms-/ & amsa & 'oscuro' \\
\hline /-mr-/ & wamra & 'niño' \\
\hline /-np-/ & anpi- & 'curar’ \\
\hline /-nt-/ & aLimanta & 'despacio' \\
\hline /-nk-/ & inkanu & 'Ingano' \\
\hline /-nč-/ & aLinči- & 'componer' \\
\hline /-ns-/ & čansama & 'más lejos' \\
\hline /-nm-/ & atunmikanki & 'ser alto' \\
\hline /-nr-/ & rinri & 'oreja' \\
\hline /-lp-/ & alpa & 'tierra' \\
\hline /-lk-/ & kulki & 'plata' \\
\hline /-rp-/ & kungurpi & 'en la rodilla' \\
\hline /-rt-/ & kungurta & 'sobre la rodilla' \\
\hline /-rk-/ & warki & 'hermano' \\
\hline /-rs-/ & pirsina- & 'santiguar' \\
\hline$/-\mathrm{jx}-/$ & kajxartin & 'este jardín' \\
\hline
\end{tabular}




\begin{tabular}{|c|c|c|}
\hline$/-\mathrm{nx}-/$ & atunxartin & 'gran jardín' \\
\hline /-rm-/ & arma- & ‘bañar’ \\
\hline /-rL-/ & warLi- & 'dañarse' \\
\hline /-jt-/ & tajta & 'padre' \\
\hline$-/ \mathrm{jč}-/$ & ajča & 'carne' \\
\hline /-jn-/ & ajni- & 'responder' \\
\hline /-js-/ & ajsa- & 'sacar' \\
\hline /-jp-/ & kajpi & 'aquí' \\
\hline /-sL-/ & saLita & 'poco' \\
\hline /-sw-/ & aswa & 'chicha' \\
\hline /-jm-/ & čajma & 'alto' \\
\hline /-jr-/ & čajra & 'todavía' \\
\hline /-lw-/ & čalwa & 'pez' \\
\hline$/-\mathrm{sj}-/$ & čisjaku & 'ya me voy' \\
\hline /-pj-/ & upja- & 'beber' \\
\hline /-jk-/ & jajku- & 'entrar' \\
\hline /-jL-/ & kajLaLa- & 'acercarse' \\
\hline /-mč/ & kamčipikanki & 'estás allá' \\
\hline /-pw-/ & karipwanki & 'buenos días' \\
\hline /-čw-/ & kičwa & 'quinchua' \\
\hline /-mn-/ & kimna- & 'vomitar' \\
\hline /-pr-/ & lipra & 'libra' \\
\hline$/-\mathrm{kr}-/$ & wakra & 'ganado' \\
\hline$/-x j-/$ & tuxja- & 'reventar' \\
\hline /-kw-/ & tukwi & 'todos' \\
\hline /-xl-/ & suxlamuča & 'un trapo' \\
\hline /-rw-/ & kunkurwa & 'con la rodilla' \\
\hline /-jñ-/ & kajñanpi & 'este camino' \\
\hline /-jl-/ & kajlamuča & 'este trapo' \\
\hline /-jw-/ & kajwamra & 'este niño' \\
\hline /-xw-/ & suxwata & 'un niño' \\
\hline /-xn-/ & suxnina & 'una candela' \\
\hline /-xñ-/ & suxñaxča & 'un peine' \\
\hline /-mw-/ & kamwa & 'contigo' \\
\hline
\end{tabular}

Hay que notar, como ya lo mencioné, que los fonemas /-n/ y /-m/ se neutralizan delante de un /p-/ explosivo. Sólo la etimología puede diferenciar pues palabras como /kampa/ y /anpi/ la pronunciación es igual.

Hay también una tendencia a la neutralización entre /-r/ y /-x/ en contacto con $/ \mathrm{k}-/$, pero no se realiza siempre como en el caso anterior. 
Sin embargo, no hay ningún ejemplo de oposición fonológica entre [-r] implosivo y $[-\mathrm{x}]$ implosivo.

\section{Vocales}

a) Iniciales: Tipos silábicos: V (inicial de palabra)

VC (inicial de palabra)

Todos los fonemas vocálicos pueden ocurrir en la inicial de la palabra tanto en sílaba abierta como en sílaba cerrada:

$\begin{array}{lc}\text { ima } & \text { /i/ } \\ \text { imapa } & \text { 'cualquier cosa' } \\ \text { inti } & \text { 'por qué?' } \\ \text { inka } & \text { 'sol' } \\ \text { iskaj } & \text { 'Inga' } \\ \text { iskun } & \text { ''os' } \\ \text { ismuska } & \text { 'nueve' } \\ & \text { 'pútrido' } \\ \text { ačika } & \text { /a/ 'varios' } \\ \text { ačka } & \text { ''largo, bastante' } \\ \text { aLanka } & \text { 'quizás' } \\ \text { alku } & \text { 'perro' } \\ \text { aLiLa } & \text { 'bien', } \\ \text { alpa } & \text { 'tierra', } \\ \text { amsa } & \text { 'oscuro' } \\ & \text { /u/ 'aji' } \\ \text { uču } & \text { 'ceniza' } \\ \text { učpa } & \text { 'pequeño' } \\ \text { učuLa } & \text { 'dentro' } \\ \text { ukupi } & \text { 'cabeza' } \\ \text { uma } & \text { 'enfermo' } \\ \text { unkux } & \text { 'piojo' } \\ \text { usa } & \text { 'hierba' } \\ \text { uxsa } & \end{array}$

b) Mediales: Tipos silábicos: CVC (en cualquier posición en la palabra). Los tres fonemas vocálicos se encuentran en esta posición y forman la parte más sonora de la sílaba:

$\begin{array}{ll}\text { aLimanta } & \text { 'despacio' } \\ \text { aLičinkapa } & \text { 'para acomodar' } \\ \text { at } u \text { n } & \text { 'alto, grande' }\end{array}$




$\begin{array}{ll}\text { čanpa } & \text { 'zanja' } \\ \text { činkwal } & \text { 'ciego, bizco' } \\ \text { čunka } & \text { 'diez' } \\ \text { Lamka- } & \text { 'tocar' } \\ \text { Lunči } & \text { 'pared' } \\ \text { miski } & \text { 'sabroso' }\end{array}$

c) Finales: Tipo silábico: CV (en cualquier posición en la palabra). Los tres fonemas vocálicos se encuentran en sílaba abierta:

$\begin{array}{ll}\text { či } & \text { 'aquel' } \\ \text { čaki } & \text { 'pié' } \\ \text { čuču } & \text { 'seno' } \\ \text { taruka } & \text { 'venado' } \\ \text { siLu } & \text { 'luna' } \\ \text { pusu } & \text { 'hueco' }\end{array}$

9.-Conclusiones:

Si se considera in abstracto el porcentaje de los fonemas vocálicos y consonánticos, las tres vocales y las 14 consonantes del Inga están en la relación 1/4,6. En el Quichua del Perú las consonantes son más numerosas, alcanzan a 26 , pero también tiene 3 vocales; la relación es pues de $1 / 8,6$.

En un texto Inga seguido, los porcentajes de vocales y consonantes son los siguientes:

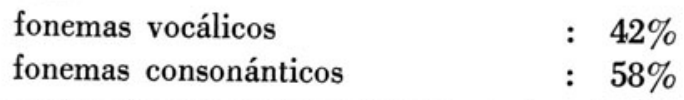

Vocales y consonantes se encuentran pues en la relación de $1 / 1,38$.

El sistema fonológico del Inga presenta en resumen las principales características siguientes:

-Tiene un sistema consonántico de 14 fonemas localizados en cuatro puntos de articulación (labial, dental, prepalatal y velar). Este sistema está organizado según dos correlaciones: una de fricción que comprende cuatro pares de sonidos que corresponden a los cuatro puntos de articulación de las oclusivas y una correlación de nasalización que sólo comprende tres pares de sonidos. Algunas oposiciones consonánticas se neutralizan en posición implosiva final de la palabra o seguida de otra consonante.

-El sistema vocálico es triangular y comprende tres vocales que no presentan ninguna neutralización.

-El sistema prosódico desconoce el uso diferenciativo de la cantidad y del acento de fuerza que sólo representa una variación extrafonológica estilística o individual. 